\title{
Albuminuria screening in children with type 1 diabetes prior to and during the COVID-19 pandemic
}

\author{
Kristen Favel $^{1 凶}$, Cherry Mammen ${ }^{1,3}$ and Constadina Panagiotopoulos ${ }^{2,3}$ \\ (c) The Author(s), under exclusive licence to the International Pediatric Research Foundation, Inc 2022
}

BACKGROUND: Albuminuria is an important risk factor for adverse renal and cardiovascular outcomes in type 1 diabetes (T1D). We sought to describe: (1) adherence to albuminuria screening prior to and during the COVID-19 pandemic and (2) occurrence of abnormal urine albumin-creatinine ratio (ACR) tests in children with T1D.

METHODS: This cohort study involved children aged 18 years or younger with T1D followed in the diabetes clinic at a pediatric tertiary center. Data was collected from 2016 to 2020. Adherence was defined by Diabetes Canada (DC) Guidelines for T1D in Children and Adolescents (2018).

RESULTS: Of the 165 children who met DC criteria for screening; 88 (32\%) were male and the median age at diagnosis was 5.8 years. Twenty-eight (17\%) children had not completed a single ACR test, and $30(18 \%)$ completed all eligible ACR tests. Test completion decreased from $66 \%$ in 2019 to $45 \%$ in 2020 . Of the 345 ACR tests completed, 40 (11\%) were abnormal (>2.5 mg/mmol) and 29 abnormal ACR tests (72\%) were not repeated.

CONCLUSION: Adherence to albuminuria screening in this pediatric diabetes clinic is suboptimal with deterioration during the COVID-19 pandemic. Patient/physician and program-level strategies to improve adherence will play an important role in quality improvement.

Pediatric Research (2022) 92:1370-1373; https://doi.org/10.1038/s41390-022-01971-7

\section{IMPACT:}

- Albuminuria screening is an important part of pediatric diabetes care.

- In our study, pediatric albuminuria screening adherence was suboptimal at $66 \%$ in 2019 and deteriorated during the pandemic to $45 \%$ in 2020.

- Program and patient-level adherence to clinical guidelines and barriers to accessing diabetes care during the pandemic merit further study.

\section{INTRODUCTION}

Albuminuria is well established as both an indicator of kidney injury, as well as a risk factor for the development of chronic kidney disease (CKD) and adverse cardiovascular outcomes in type 1 diabetes (T1D). The clinical features of diabetic kidney disease (DKD) including impaired glomerular filtration rate (GFR), hypertension, and albuminuria may become apparent during childhood. Reported albuminuria prevalence ranges between 1 to $10 \%$ in children and youth with T1D. ${ }^{1-3}$ Pediatric T1D clinical practice guidelines recommend screening for CKD using spot urine albumin-to-creatinine ratio (ACR) to detect albuminuria., ${ }^{4,5}$ Diabetes Canada recommends that children $\geq 12$ years with diabetes duration $>5$ years should be screened annually for CKD with a first morning urine ACR or a random urine ACR. ${ }^{4}$

Although a significant proportion of individuals with T1D are diagnosed and receive care during childhood, literature on process-of-care indicators, including number of physician visits,
A1C tests, and screening assessments for diabetes-related complications have shown that children with diabetes are not receiving optimal care per national clinical practice guidelines. In self-reported surveys and administrative data studies, several factors associated with suboptimal adherence were identified, including longer duration of diabetes, transitional care in adolescents, and lower family income and socioeconomic status., ${ }^{6,7}$ Compounding the aforementioned sociodemographic factors, medical care across all age groups has been greatly impacted by the COVID-19 pandemic. Although telemedicine models for the ambulatory care of children with diabetes have been examined with optimistic results, ${ }^{8-10}$ the impact of workforce redeployment and the large-scale, abrupt shift to virtual care in many clinical settings has undoubtedly impacted quality of care and access to care. Especially in the context of the paradigm shift of the pandemic, analyzing and optimizing the quality of delivered care is an essential undertaking. In this study, we describe (1)

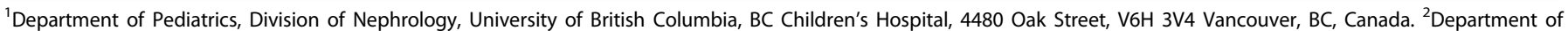

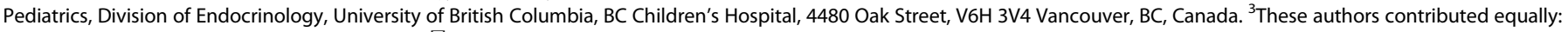
Cherry Mammen, Constadina Panagiotopoulos. ${ }^{凶}$ email: Kristen.Favel@cw.bc.ca 


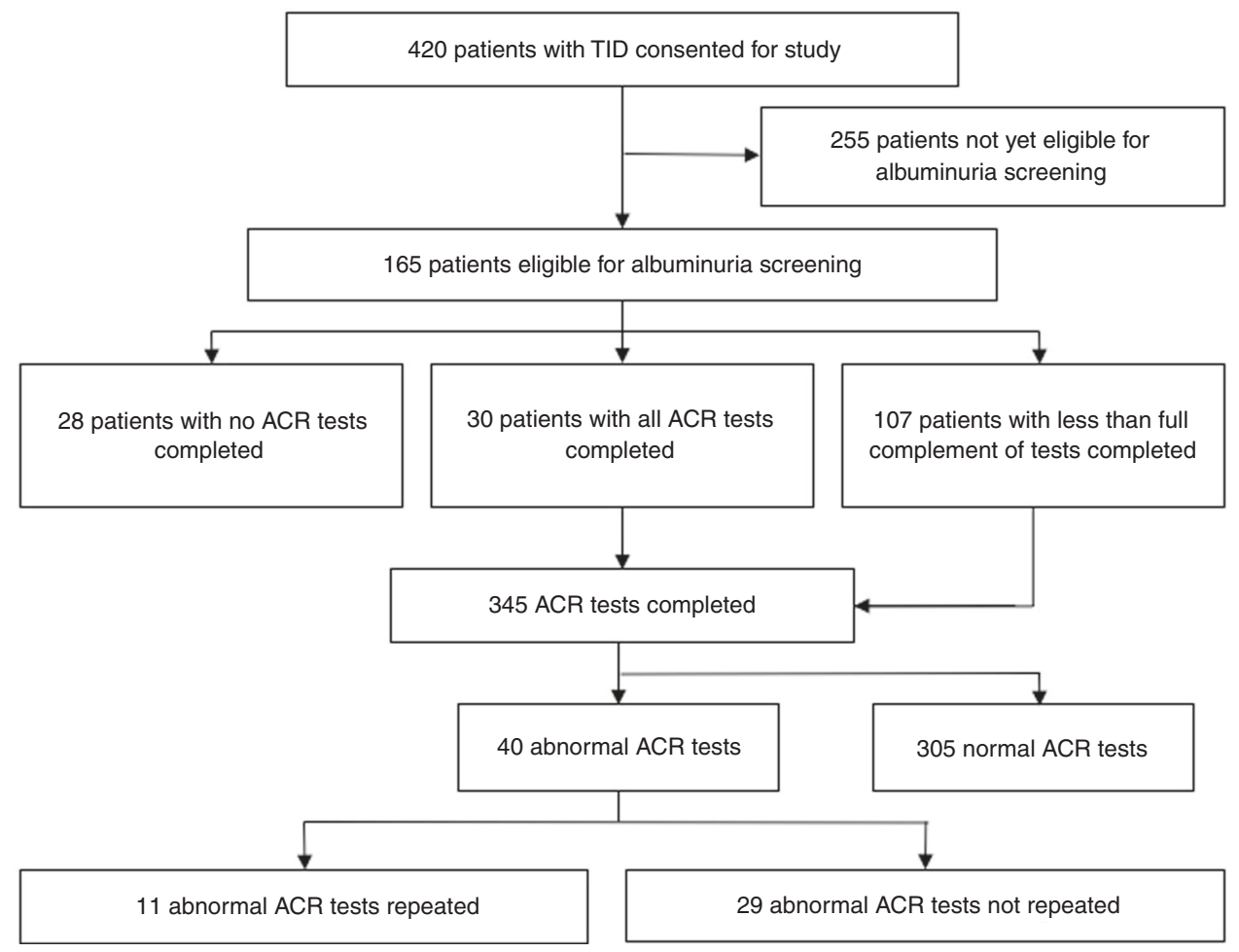

Fig. 1 Flow diagram for study participants and aggregated urine ACR results (abnormal urine ACR defined as value greater than $2.5 \mathrm{mg} / \mathrm{mmol}$ ).

adherence to albuminuria screening and (2) occurrence of abnormal urine ACR tests in a cohort of children with type 1 diabetes prior to and during the COVID-19 pandemic.

\section{METHODS}

\section{Study population and design}

An ongoing ambispective cohort study began in 2016 to assess the prevalence of abnormalities in estimated glomerular filtration rate in children with T1D, including children with a history of concomitant diabetic ketoacidosis and acute kidney injury. ${ }^{11}$ Study patients were identified and recruited through the T1D clinic between December 2016 to February 2019. They were eligible for inclusion if they were 18 years of age or younger and were followed in the British Columbia Children's Hospital (BCCH) T1D clinic. They consented to retrospective and prospective review of their medical records. All data collection occurred between December 2016 to January 2021.

This study was conducted at BCCH in Vancouver, BC, Canada. Prior to March 2020, all children followed in the T1D clinic were seen in-person. A state of emergency was declared on March 18, 2020 in relation to the COVID-19 pandemic. In the T1D clinic, all routine outpatient follow-up visits were transitioned from in-person to virtual/telephone attendance as of March 23, 2020 and continued for the duration of this study. The University of British Columbia Clinical Research Ethics Board reviewed and approved the study protocol.

\section{Data collection}

The study team reviewed paper and electronic medical records. Data pertaining to demographic characteristics, medical history, and clinical characteristics related to diabetes care and outpatient laboratory parameters were collected including clinic date, height, weight, blood pressure, and hemoglobin $\mathrm{A} 1 \mathrm{C}(\mathrm{A} 1 \mathrm{C})$ paired with outpatient serum creatinine and urine ACR results. Year of birth and age at diagnosis of diabetes were used to ascertain the year each enrolled individual was eligible to initiate albuminuria screening. All spot urine ACR tests completed between January 1 to December 31 in the year the patient turned 12 years of age and each subsequent year up to December 31, 2020 were captured. Urine ACR tests done in the calendar year prior to patients becoming eligible were also captured. All A1C values from the year of initial eligibility were recorded for each patient.

\section{Definitions}

Based on Diabetes Canada guidelines, urine ACR was categorized as abnormal for values greater than $2.5 \mathrm{mg} / \mathrm{mmol}$, and albuminuria was diagnosed on the basis of two consecutive first morning urine ACR tests obtained at 3- to 4- month intervals over a 6- to 12 - month period. ${ }^{4}$

\section{Statistical analysis}

Descriptive data was reported as mean and standard deviation for normally distributed continuous variables, median and interquartile range for non-normally distributed continuous variables, and frequencies and percentages for categorical variables. Normality of variables was tested using the Shapiro-Wilk test. Comparisons between means were done with the Student's $t$-test or ANOVA for normally distributed continuous variables, Wilcoxon tests or Kruskal-Wallis test for non-normally distributed continuous variables, and with the $x^{2}$ test for categorical variables. A $p$ value less than 0.05 was considered significant. Statistical analyses were performed using $\mathrm{R}$ version 4.0.2.

\section{RESULTS}

As of December 31, 2020, 420 patients were consented for participation in the study assessing the prevalence of abnormalities in eGFR, of which 165 patients (39\%) had become eligible to initiate annual albuminuria screening (see Fig. 1). Table 1 highlights the demographic and clinical characteristics of the eligible cohort.

\section{Test completion by individual}

The period of eligibility for initiation of albuminuria screening spanned from 2014 to 2020. Eighty-three percent (137/165) of patients completed at least one ACR test. Eighteen percent of patients (30/165) completed all eligible ACR tests, and 65\% (107/ 165) completed less than their total complement of ACR tests, and 
Table 1. Demographic characteristics of children eligible to start annual albuminuria screening.

\begin{tabular}{|l|l|}
\hline Characteristics & $\begin{array}{l}\text { Eligible cohort }(\boldsymbol{n}= \\
\mathbf{1 6 5})\end{array}$ \\
\hline Male & $88(53)$ \\
\hline Insulin pump & $85(51)$ \\
\hline$\geq 2$ daily insulin injections & $80(49)$ \\
\hline $\begin{array}{l}\text { Duration of T1D at initiation of screening } \\
\text { (years) }\end{array}$ & $6.3[5.0 ; 8.6]$ \\
\hline Age at diagnosis (years) & $5.8[3.4 ; 8.2]$ \\
\hline A1C at initiation of screening (\%) & $8.0[7.3 ; 8.9]$ \\
\hline
\end{tabular}

Median [interquartile range] reported for quantitative variables and absolute (\%) for qualitative variables. T1D type 1 diabetes, A1C hemoglobin A1C.

$17 \%(28 / 165)$ did not complete any ACR tests over the entire period of eligibility. Of those patients who had completed all ACR tests or some lesser complement of tests, there was a higher proportion using insulin pumps (53\% and $56 \%$ respectively), as compared to those patients with no recorded ACR tests (32\%) ( $p$ $=0.08$ ). Patients who had completed all ACR tests had a slightly lower median $\mathrm{A} 1 \mathrm{C}$ at $7.8 \%[6.9 ; 8.6]$ compared to those with a lesser complement of tests or no recorded ACR tests (8.0\% [7.4; $8.9]$ and $8.1 \%[7.3 ; 9.1]$ respectively) $(p=0.3)$. Overall, there were no statistically significant differences in patient sex, insulin regimen, duration of $\mathrm{T} 1 \mathrm{D}$, age at diagnosis, or $\mathrm{A} 1 \mathrm{C}$ based on the proportion of ACR tests completed.

\section{Test completion by calendar year}

Annual urine ACR test completion decreased from $66 \%$ in the calendar year of $2019-2020$ to $45 \%$ in $2020-2021$. The latter time period includes the onset of the provincial state of emergency and transition from in-person visits to virtual/phone visits. Of those patients who had not completed any ACR tests, $57 \%(16 / 28)$ had become eligible to initiate albuminuria screening in 2020; however, this only makes up $17 \%(16 / 91)$ of patients who did not complete an ACR test in 2020.

\section{Confirmation of abnormal results}

A total of 345 ACR tests were completed for 137 patients, of which $11 \%(40 / 345)$ were abnormal (ACR $>2.5 \mathrm{mg} / \mathrm{mmol}$ ). The results of the abnormal ACR tests ranged from 3.0 to $91.5 \mathrm{mg} / \mathrm{mmol}$, with a median urine ACR of $7.9[4.1 ; 17.9] \mathrm{mg} / \mathrm{mmol}$. Sixty-five percent $(26 / 40)$ of results were between 3 to $10 \mathrm{mg} / \mathrm{mmol}$, and $35 \%$ $(14 / 40)$ of results greater than $10 \mathrm{mg} / \mathrm{mmol}$. Twenty-eight percent $(11 / 40)$ of the abnormal tests were repeated within six months of the initial result; the initial abnormal results ranged from 3.3 to 25 $\mathrm{mg} / \mathrm{mmol}$, with a median urine ACR of 4.7 [4.0; 10.0$] \mathrm{mg} / \mathrm{mmol}$, and all repeated ACR tests were normal. The remaining 72\% (29/ 40) of abnormal tests were not repeated, with the subsequent ACR test completed at least twelve months after the initial abnormal result.

\section{DISCUSSION}

In this study, we report three key findings. First, adherence to albuminuria screening recommendations was suboptimal in a cohort of pediatric patients with T1D followed at the only tertiary/ quaternary care pediatric hospital in British Columbia, Canada; specifically, only $18 \%$ of patients completing all recommended urine ACR tests, and $82 \%$ of patients having less than the full complement or no tests completed. Secondly, adherence declined in the twelve-month period from January to December 2020 coinciding with the COVID-19 pandemic. Finally, this study also demonstrated deficits in the confirmation of abnormal results, with repetition of only $28 \%$ of abnormal tests.

Adherence to recommendations for diabetes management has been highlighted as an area for improvement. In a Canadian population-based administrative study of children with T1D, it was noted that only $39 \%$ of all annual records met the criteria for optimal or good adherence to clinical practice guidelines as defined by the number of physician visits, A1C tests, glucagon prescriptions dispensed, and appropriate screening tests for diabetes-related comorbidities and complications. ${ }^{6}$ Survey data from SEARCH for Diabetes in Youth study participants similarly indicated less than optimal adherence to guidelines with less than $83 \%$ of participants reporting completion of kidney function tests, and less than $70 \%$ of participants reporting regular A1C testing and eye examination. ${ }^{7}$ While literature on patient-level adherence to annual urine ACR testing in pediatric T1D is lacking, more robust data is derived from adult T2D cohorts. Our pediatric cohort is comparable to adult cohorts, with completion of albuminuria screening in $45 \%$ to $78 \%$ of patients in three different international T2D study groups (GIANTT, TRIAD, and GUIDANCE) observed over a 12-month period or calendar year prior to $2020 .^{12-15}$ Repetition of abnormal tests is similarly suboptimal in adults with T2D with a $37 \%$ compliance rate for repeat urine ACR samples in a single-center study. ${ }^{16}$ Although the prevalence of albuminuria in children with T1D is low compared to adults, there are consequences to screening test non-adherence. At the population-level, underestimation of the prevalence of albuminuria may impact distribution of resources for medical care (i.e., referral to pediatric nephrology) and research. On a patient level, incomplete assessment of an individual's risk for a disease or related complication may delay appropriate management. Beyond early diagnosis of diabetes-related complications, process-of-care indicators like annual urine ACR tests are associated with patient satisfaction measures and self-rated quality of diabetes care. ${ }^{13}$

Adherence to medical management of chronic conditions depends on many different factors. On a patient level, there may be psychosocial and financial barriers preventing access to transportation to appointments and other healthcare facilities, or access to email or phone as a means to communicate with healthcare team members. There may also be literacy and language barriers leading to a lack of understanding of the significance of the test and instructions for conducting the test. On a healthcare provider and program level, there may be deficits in reminders for ordering tests and distribution of requisitions, where this may require navigation between paper and electronic medical records. Healthcare providers may vary in their interpretation or implementation of clinical guidelines, depending on the individual or type of provider.

In addition to the abovementioned factors, the impact of the COVID-19 pandemic on patient access to healthcare cannot be understated. With the transition to virtual care across many jurisdictions, comprehensive clinical assessment has been limited, with the omission of important physical assessments including biannual screening for hypertension. Opportunities for laboratory tests to be completed on the same day as clinics in hospitalaffiliated laboratories were reduced. Additionally, to comply with British Columbia Centre for Disease Control recommendations for indoor occupancy, access to external community laboratories was limited to appointment-only rather than walk-in access. Beyond infrastructure changes, redistribution of resources and hesitancy to access care for fear of contracting COVID-19 from contacts at hospitals, laboratories, and clinics has impacted patient access to healthcare. ${ }^{17,18} \mathrm{~A}$ decline in screening tests during the pandemic has been noted in other areas, most prominently in cancer care with more than $50 \%$ reduction across all screening tests at the peak of the pandemic in April 2020. ${ }^{19,20}$ Quality improvement in this area takes on greater importance with the ongoing use of virtual care. Without corresponding physical assessment and 
laboratory investigations, the clinical picture is incomplete, especially in the case of a condition like DKD. Strategies to improve verbal communication with patients and families and coordinate with healthcare team members will be necessary to optimally care for patients given this new paradigm.

A challenge in quality assessment of test adherence is the choice of observation period as annual tests have a lower likelihood of falling within a fixed observation period. The choice of a 12-month observation period in our study may have contributed to an underestimation of the proportion of completed tests for a particular year. Our study is limited to patients and healthcare providers affiliated with the diabetes clinic at our center, where diabetes care is also provided by subspecialists and pediatricians practicing in the community setting. Assessment of albuminuria screening adherence in alternative practice settings would be a valuable addition to our current data. Our study is also purely quantitative, where quality assessment is enhanced with the addition of qualitative data from involved parties. The development of quality improvement initiatives in albuminuria screening adherence will most certainly require the input of patients, families, and healthcare team members.

Patient and program-level strategies to improve adherence to albuminuria screening will play an important role in quality improvement in this area. The results of this study prompted our health care team to create an educational handout for patients and caregivers. The handout details the rationale and procedure for annual albuminuria screening. It is freely accessible on the $\mathrm{BCCH}$ Endocrinology and Diabetes website (http://www. bcchildrens.ca/endocrinology-diabetes-site/documents/uacr.pdf), and it will be distributed to patients and caregivers along with the urine ACR test requisition. Other interventions include review of the electronic health record to ensure appropriate reminders and prompts are in place for follow-up of abnormal tests. Future quality improvement research will assess the impact of these interventions on annual albuminuria screening adherence. Qualitative analysis of patient and caregiver barriers to accessing care merits further study and will play an important role in quality improvement in this area.

\section{REFERENCES}

1. Kahkoska, A. R. et al. The early natural history of albuminuria in young adults with youth-onset type 1 and type 2 diabetes. J. Diabet. Complications 32, 1160-1168 (2018).

2. Maahs, D. M. et al. Higher prevalence of elevated albumin excretion in youth with type 2 than type 1 diabetes: the SEARCH for diabetes in youth study. Diabetes Care 30, 2593-2598 (2017).

3. Li, L., Jick, S., Breitenstein, S. \& Michel, A. Prevalence of diabetes and diabetic nephropathy in a large U.S. commercially insured pediatric population, 2002-2013. Diabetes Care 39, 278-284 (2016).

4. Wherrett, D. K. et al. Diabetes Canada 2018 clinical practice guidelines for the prevention and management of diabetes in Canada: type I diabetes in children and adolescents. Can. J. Diabetes 42, S234-S246 (2018).

5. Chiang, J. L. et al. Type 1 diabetes in children and adolescents: a position statement by the American diabetes Association. Diabetes Care 41, 2026-2044 (2018).

6. Amed, S. et al. Adherence to clinical practice guidelines in the management of children, youth, and young adults with type 1 diabetes-a prospective population cohort study. J. Pediatr. 163, 543-548 (2013).

7. Waitzfelder, B. et al. Adherence to guidelines for youths with diabetes mellitus. Pediatrics 128, 531-538 (2011).

8. Wood, C. L. et al. Use of telemedicine to improve adherence to American Diabetes Association standards in pediatric type 1 diabetes. Diabetes Technol. Ther. 18, 7-14 (2016).

9. Raymond, J. K. Models of care for adolescents and young adults with type 1 diabetes in transition: shared medical appointments and telemedicine. Pediatr. Ann. 46, e193-e197 (2017).

10. Reid, M. W. et al. CoYoT1 clinic: home telemedicine increases young adult engagement in diabetes care. Diabetes Technol. Ther. 20, 370-379 (2018).
11. Favel, K., Mammen, C. \& Panagiotopoulos, C. Chronic kidney disease prevalence and glomerular filtration rate trends in children with type 1 diabetes. J. Endocr. Soc. 5, A457-A458 (2021).

12. Sidorenkov, G., Haaijer-Ruskamp, F. M., de Zeeuw, D. \& Denig, P. A longitudinal study examining adherence to guidelines in diabetes care according to different definitions of adequacy and timeliness. PLOS ONE 6, e24278 (2011).

13. Ackermann, R. T. et al. Is the number of documented diabetes process-of-care indicators associated with cardiometabolic risk factor levels, patient satisfaction, or self-rated quality of diabetes care? The Translating Research into Action for Diabetes (TRIAD) study. Diabetes Care 29, 2108-2113 (2006). Translating Research into Action for Diabetes (TRIAD) Study Group.

14. Stone, M. A. et al. Quality of care of people with type 2 diabetes in eight European countries: findings from the Guideline Adherence to Enhance Care (GUIDANCE) study. Diabetes Care 36, 2628-2638 (2013). GUIDANCE Study Group.

15. Hellemons, M. E., Denig, P., de Zeeuw, D. \& Voorham, J. Lambers Heerspink, H.J. Is albuminuria screening and treatment optimal in patients with type 2 diabetes in primary care? Observational data of the GIANTT cohort. Nephrol. Dial. Transplant. 28, 706-715 (2013)

16. Baskar, V., Kamalakannan, D., Holland, M. R., Catchpole, C. R. \& Singh, B. M. Uncertain clinical utility of contemporary strategies for microalbuminuria testing. Diabetes Obes. Metab. 5, 262-266 (2003).

17. Lazzerini, M. et al. Delayed access or provision of care in Italy resulting from fear of COVID-19. Lancet Child Adolesc. Health 4, e10-e11 (2020).

18. Jeffery, M. M. et al. Trends in emergency department visits and hospital admissions in health care systems in 5 states in the first months of the COVID-19 pandemic in the US. JAMA Intern. Med. 180, 1328-1333 (2020).

19. Patt, D. et al. Impact of COVID-19 on cancer care: how the pandemic is delaying cancer diagnosis and treatment for American Seniors. JCO Clin. Cancer Inform. 4, 1059-1071 (2020).

20. Bakouny, Z. et al. Cancer screening tests and cancer diagnoses during the COVID19 pandemic. JAMA Oncol. 7, 458-460 (2021).

\section{ACKNOWLEDGEMENTS}

We thank the research staff at the BCCH Research Institute for their assistance in the administration of this study per the Ethical Conduct for Research Involving Humans.

\section{AUTHOR CONTRIBUTIONS}

The authors confirm contribution to the paper as follows: study conception and design (K.F., C.P., and C.M.), data collection (K.F.), analysis and interpretation of results (K.F., C.P., and C.M.), and draft manuscript preparation (K.F., C.P., and C.M.). All authors reviewed the results and approved the results and approved the final version of the manuscript.

\section{FUNDING}

The research was funded in part by the Canucks for Kids Fund (C.P.). KF also received fellowship training support from the University of British Columbia Clinician Investigator Program. These funding sources had no role in the design and conduct of the study.

\section{COMPETING INTERESTS}

The authors declare no competing interests.

\section{CONSENT TO PARTICIPATE}

Each subject was adequately informed of the aims, methods, sources of funding, any possible conflicts of interest, institutional affiliations of the research team, the benefits and potential risks of the study and the discomfort it may entail, and any other relevant aspects of the study.

\section{ADDITIONAL INFORMATION}

Correspondence and requests for materials should be addressed to Kristen Favel.

Reprints and permission information is available at http://www.nature.com/ reprints

Publisher's note Springer Nature remains neutral with regard to jurisdictional claims in published maps and institutional affiliations. 